\title{
Point-by-point inscription of 250-nm-period structure in bulk fused silica by tightly-focused femtosecond UV pulses: experiment and numerical modeling
}

\author{
David N. Nikogosyan ${ }^{1,2 *}$, Myhaylo Dubov ${ }^{1}$, Holger Schmitz ${ }^{1}$, Vladimir Mezentsev ${ }^{1}$, \\ Ian Bennion ${ }^{1}$, Padraig Bolger ${ }^{3}$ and Anatoly V. Zayats ${ }^{3}$ \\ ${ }^{1}$ Photonics Research Group, Aston University, Birmingham B4 7ET, United Kingdom \\ ${ }^{2}$ Physics Department, University College Cork, Cork, Ireland, e-mail: niko@phys.ucc.ie \\ ${ }^{3}$ Centre for Nanostructure Media, IRCEP, The Queen's University of Belfast, Belfast BT7 1NN, \\ United Kingdom
}

\begin{abstract}
By conducting point-by-point inscription in a continuously moving slab of a pure fused silica at the optimal depth $(170 \mu \mathrm{m}$ depth below the surface), we have fabricated a 250 -nm-period nanostructure with $30 \mathrm{~nJ}, 300 \mathrm{fs}, 1 \mathrm{kHz}$ pulses from frequency-tripled Ti:sapphire laser. This is the smallest value for the inscribed period yet reported, and has been achieved with radical improvement in the quality of the inscribed nanostructures in comparison with previous reports. The performed numerical modeling confirms the obtained experimental results.
\end{abstract}

Keywords: femtosecond UV laser, microfabrication, fused silica, nanostructure, differential interference contrast microscopy, atomic-force microscopy

PACS code numbers: 42.79.Dj; 42.81.-i; 81.07.-b; 81.16.-c

\section{Introduction}

In 2004, two groups independently reported the application of a point-by-point ( $\mathrm{PbP})$ technique to fibre Bragg grating (FBG) inscription in standard non-photosensitive fibers [1-3]. Both groups used tightly-focussed femtosecond (fs) IR laser $(\lambda=800 \mathrm{~nm})$ radiation and a submicron precision positioning system. The FBGs produced exhibited either fourth-order $(\Lambda=2.14$ $\mu \mathrm{m}$ ) [1] or from first- to fourth-order (with the strongest second-order, $\Lambda=1.07 \mu \mathrm{m}$ ) [2,3] periods for the reflection peak at the telecommunications wavelength $1.55 \mu \mathrm{m}$. Since that time, 
the $\mathrm{PbP}$ technique employing the output of a femtosecond Ti:sapphire laser has evolved to become a relatively routine method for the fabrication of gratings in fibers with typical values of the inscribed period of $1.07 \mu \mathrm{m}$ [3-8] or $1.12 \mu \mathrm{m}$ (third-order grating for the $1080 \mathrm{~nm}$ reflection) [9]. Recently, with tightly-focused $800 \mathrm{~nm}$ femtosecond light pulses, injected into a slab of pure fused silica using a special, reflective microobjective, the fabrication of first-order gratings for $1550 \mathrm{~nm}$ wavelength $(\Lambda=0.535 \mu \mathrm{m})$ was reported [10,11]. Very recently, by introducing indexmatching fluid between the planar microscope cover slip and fiber, the same $535 \mathrm{~nm}$ period was achieved with FBG recording in standard fiber [12].

It should be noted that all of the foregoing investigations on point-by-point microfabrication have employed $800 \mathrm{~nm}$ femtosecond light pulses, which excite the samples of fused silica (or germanosilicate glass) via five-photon absorption [13]. Such a multi-photon approach can employ the different wavelengths and different numbers of photons in one elementary absorbing act [14], so facilitating inscription inside various non-photosensitive optical materials. It is also known that the propagation of a femtosecond $800 \mathrm{~nm}$ pulse inside a bulk dielectric (e.g. fused silica glass) with a peak power exceeding the threshold (critical power) results in self-focusing. Remarkably, this regime is characterized by reduction of the spatial dimensions of the photoinduced material modifications below the diffraction limit [15]. Since the achieved size of the pitch (modification) is about $270 \mathrm{~nm}$ and much smaller than the inscribing wavelength of 800 $\mathrm{nm}$, further feature size reduction would appear to be highly unlikely using this method. However, shifting the wavelength of the inscribing Ti:sapphire laser radiation into the UV range (e.g. to $267 \mathrm{~nm}$ with simultaneous decrease in the order of absorption process from five-photon to two-photon [14]) immediately makes it possible to record structures with even smaller periods. Such a development is very important, for example, for the point-by-point fabrication of first-order Bragg gratings possessing a peak reflectance wavelength of $\sim 1 \mu \mathrm{m}$. 
In our previous work, the use of $267 \mathrm{~nm}$ femtosecond pulses with $82 \mathrm{~nJ}$ energies led us to the inscription of 300-nm-period structures [16]. In the current work, the optimising of the inscription depth has allowed us to decrease the inscription energy down to $30 \mathrm{~nJ}$ and to inscribe the 250-nm-period structure. In addition, the quality of the nanostructures was significantly improved in comparison with our previous report.

\section{Experimental set-up}

Femtosecond pulses at $800 \mathrm{~nm}$ were produced by a Ti:sapphire chirped pulse amplification laser system consisting of a „Tsunami“ oscillator and a „Spitfire“ amplifier (both from SpectraPhysics). The laser system delivered $0.8 \mathrm{~mJ}$ pulses with $150 \mathrm{fs}$ duration and $1 \mathrm{kHz}$ repetition rate. The IR beam diameter after the amplifier was $2.5 \mathrm{~mm}$ at FWHM. The set-up for thirdharmonic generation (THG) was similar to that described earlier [17]. The pulses at $267 \mathrm{~nm}$ were produced by non-collinear sum-frequency mixing between fundamental radiation and that at the second harmonic (Fig. 1 a). A half-wave plate was used to distribute the energy of $800 \mathrm{~nm}$ pulses between two channels, which allowed us to manipulate the energy at the entrance of second-harmonic generator and, thus, the energy of the output radiation at $267 \mathrm{~nm}$. The pulses at $400 \mathrm{~nm}$ were produced in a $1 \mathrm{~mm}$ thick BBO crystal cut for type I collinear second harmonic generation $\left(\theta=29.2^{\circ}, \varphi=90^{\circ}\right)$ [18]. A second half-wave plate was used for $90^{\circ}$ polarization rotation of the $400 \mathrm{~nm}$ beam. Using three mirrors with high reflectance at $400 \mathrm{~nm}$, the second harmonic beam was separated from the fundamental. The 400 and $800 \mathrm{~nm}$ pulses were directed into a $1.0 \mathrm{~mm}$ thick BBO crystal cut for type I sum-frequency generation $\left(\theta=44.3^{\circ}, \varphi=90^{\circ}\right)$ [18]. The angle between the $800 \mathrm{~nm}$ and $400 \mathrm{~nm}$ beams was less than $2^{\circ}$ in the horizontal plane. Using highly-reflecting UV mirrors and the non-collinear geometry of THG, we easily separated the $267 \mathrm{~nm}$ radiation. The UV pulse energy was monitored by a PD10 photodiode (Ophir Optronics). The energy of the third harmonic pulses was about $80 \mu \mathrm{J}$ with pump energy at 800 
$\mathrm{nm}$ of $800 \mu \mathrm{J}$. By varying the optical delay and measuring the cross-correlation function between the fundamental pulse and it's second harmonic, we estimated the width of both $400 \mathrm{~nm}$ and 267 nm pulse to be about 300 fs.

Fused silica samples of $50 \times 20 \mathrm{~mm}$ size and $1 \mathrm{~mm}$ thickness (Schott Glas) were used in the experiments. They were moved in the horizontal plane in two perpendicular directions by an airbearing translation stage ABL-1000 (Aerotech). The translation speed was varied in the range of $0.25-1.0 \mathrm{~mm} / \mathrm{s}$. The absolute and relative micropositioning accuracies were both better than 50 nm.

The UV laser beam was directed in a strictly perpendicular direction on to the surface of the fused silica sample from the top (Fig. 1 b). It could be focused to any selected depth between 0 and $600 \mu \mathrm{m}$ below the surface with accuracy of $1 \mu \mathrm{m}$. For focusing, we used a reflective microscope objective with numerical aperture of 0.65 (Ealing), manipulated by a 3Dmicropositioning manual translation stage 17 MAX 303 (Melles Griot). The inscription energy values were varied between 20 and $400 \mathrm{~nJ}$, whilst the length of inscribed tracks was usually between 0.5 and $4 \mathrm{~cm}$.

\section{Characterisation of inscribed structures}

To establish the optimal conditions for inscription, one should carefully adjust at least four parameters including, particularly, the laser pulse energy, the speed of translation stage movement, the focus depth inside the sample, and the polarization of the inscribing light with respect to the direction of sample movement. Other parameters, including the numerical aperture of the microscope objective, the repetition rate, the wavelength and the duration of the inscribing pulses, also could be varied. The resulting number of experimental tracks could easily reach some thousands: thus, an express visualization method is needed for such optimisation. 
We perform the characterisation of the irradiated samples using an optical microscope, Axioscope-2 MOT plus (Zeiss), which was equipped for both transmitted light and differential interference contrast (DIC) measurements. The resolution of a conventional optical microscope is considered to be of the order of the illumination wavelength: in our experiment, even with the use of a blue filter it was rather difficult to distinguish the 600-nm-period perturbations induced in the bulk of the material. However, the use of DIC technique enabled us to monitor structures with periods down to $250 \mathrm{~nm}$. In the experiments, we used the combination of a PlanApochromat oil immersed objective (×100/1.40/DIC) and an Achromatic-Aplanatic condenser (1.4H/PH/DIC) with numerical aperture of 0.6 (or higher). A DIC-prism (III/1.4) and DIC-slider $(\times 100 / 1.40$ III $)$ were also used; such a combination seems to be the best one commercially available from Zeiss. The refractive index variation is expected to be of the order of $10^{-4}$, which corresponds to a few times more than the sensitivity level of this DIC microscope, based on the comparable experiments conducted with known samples.

It is important to note that DIC microscopy does not present the real image; rather, the resulting picture contains the information of both the intensity distribution and the derivative of the optical phase between two orthogonally polarised beams, spatially separated by a distance smaller than the resolution of the $\times 100, \mathrm{NA}=1.4$ microscope objective. Thus, periods of about few hundred nanometres can be detected.

For the independent resolution check of DIC microscopy, some tracks exhibiting topographic changes of the sample surface were characterized with atomic-force microscopy (AFM) [19]. We used a commercial instrument, the Dimension Nanoscope III (VEECO), working in the tapping mode.

\section{Results and discussion}


We allied the optimisation procedure to the search for the optimal focus depth whereby inscription with smaller energy (leading to smaller beam diameter and, hence, to smaller nanostructure period) will be possible. In contrast with [16], in this work we used only the $\pi$ polarization of the $267 \mathrm{~nm}$ inscribing beam (parallel to the translation direction).

We started our inscription experiments by focussing our microscope objective on the surface of the fused silica sample. With pulse energy as small as $38 \mathrm{~nJ}$, we were able to record nanostructures with a translation speed of $1.0 \mathrm{~mm} / \mathrm{s}$. Taking into account the repetition rate of 1 $\mathrm{kHz}$, it is easy to deduce that the recorded grating nanostructure possesses a $1000 \mathrm{~nm}$ period (Fig. 2). The DIC microphotography taken at the surface of our fused silica sample shows that the laser beam cross-section in the focal plane is not ideal and varies in size from pulse to pulse. A similar picture was revealed by topographic changes of the sample surface while using the AFM technique (Fig. 3 a). The asymmetry seen in individual voxels is probably related to the light diffraction on wire holders of a small mirror inside the microobjective. From further consideration it will be evident that such diffraction becomes unimportant while focussing inside the slab of fused silica. We have also applied the AFM method for the independent calibration of our DIC microscopy approach. Figure $3 \mathrm{~b}$ (cross-section of the image of Fig. 3 a along the grating) shows that 10 periods of our nanostructure inscribed on the surface of fused silica sample correspond exactly to $10 \mu \mathrm{m}$ length, confirming our calibration shown at Fig. 2 (the scale bar is $10 \mu \mathrm{m}$ ). The cross-section in the perpendicular direction (presented in Fig. $3 \mathrm{c}$ ), which is an ablation profile, gives an upper estimate of $400 \mathrm{~nm}$ for the diameter of the laser beam crosssection at FWHM at the surface of our sample, which agrees with the spot sizes deduced from Fig. $2(210-310 \mathrm{~nm})$.

To estimate the beam-waist diameter in the focal plane, one can use the well-known expression for diffraction-limited focusing, $w_{0}=C \lambda / N A$, where $\lambda$ is the inscription wavelength, $C$ is a constant $\sim 1.2-1.6$, defined by the exact profile of the laser beam, and $N A$ is the numerical 
aperture of the microscope objective. Substituting $\lambda=267 \mathrm{~nm}$ and $N A=0.65$ gives the beamwaist diameter $w_{0}$ of 500-600 nm, which agrees with the experimental values given above.

Much better results - inscription at smaller translation speed values down to $0.25 \mathrm{~mm} / \mathrm{s}$ - were obtained with the inscribing light tightly focussed to a depth of $170 \mu \mathrm{m}$ below the surface of a fused silica sample. Figure 4 a demonstrates the periodic structures obtained using $30 \mathrm{~nJ}$ pulses with sample translation speed values of $1.0,0.5$, and $0.4 \mathrm{~mm} / \mathrm{s}$, respectively, i.e. with periods 1000, 500 and $400 \mathrm{~nm}$, respectively. The excellent quality of the gratings obtained should be emphasized ( $c f$. structures with similar periods obtained in our previous work and depicted in Fig. 4 b). Furthermore, the irregularity in spot size was strongly reduced.

The measurements of the beam-waist diameter of the spots presented in Fig. 4 a show that the decrease of the translation speed value from $1.0 \mathrm{~mm} / \mathrm{s}$ to $0.5 \mathrm{~mm} / \mathrm{s}$ and further to $0.4 \mathrm{~mm} / \mathrm{s} \mathrm{leads}$ to the simultaneous decrease of the beam-waist diameter from $630 \mathrm{~nm}$ to $300 \mathrm{~nm}$ and then to 230 nm (with accuracy $\pm 10 \%$ ). Interestingly, a similar feature could be seen in the tracks with similar periods presented in our previous report (Fig. 4 b). This could be related to the change of glass properties (e.g., induced UV absorption and/or refractive index change) performed by the previous neighbour inscription pulse (pulses), which increases the absorption and hence following self-focusing for the next inscription pulse (pulses). The decrease of the translation speed value should increase the probability of such effect.

Figure 5 shows the nanostructures obtained at the same focus depth $(170 \mu \mathrm{m})$ using sample translation speed values 0.3 and $0.25 \mathrm{~mm} / \mathrm{s}$, i.e. with periods of 300 and $250 \mathrm{~nm}$, respectively. The deterioration of quality of these gratings could be connected with overlapping between neighbouring spots (if the size of a spot exceeds a half of the nanostructure period, i.e., $150 \mathrm{~nm}$ ) and/or with the nanostructure period smaller than the resolution limit of DIC microscopy. Future investigation of recorded structures by scanning electron microscopy would help to elucidate this point. In any case, in this work at optimal focus depth, we have obtained evidence for 
nanostructure inscription with a period as small as $250 \mathrm{~nm}$. We recall that, in our previous report [16], for this particular polarization of the inscribing laser beam only the 400-nm-period structure was recorded.

The importance of using the optimal focus depth can be illustrated by experiments conducted at $330 \mu \mathrm{m}$ below the sample surface. At this focus depth, using $89 \mathrm{~nJ}$ pulses, we managed to record structures with periods down to $500 \mathrm{~nm}$ (Fig. 6); at the optimal inscription depth (170 $\mu \mathrm{m}$ ), with $92 \mathrm{~nJ}$ pulses, we recorded the structures of 400- and 300-nm periods (Fig. 7).

It should be emphasized that the value of the optimal inscription depth obtained in this work $(170 \mu \mathrm{m})$, differs from the one obtained in our previous report $(300 \mu \mathrm{m})$. The reason for this could be the inaccurate positioning of the correction ring of the microscope objective. Due to this, in our previous work, the microscope objective was not aligned for minimisation of aberrations whilst being focussed on the surface of the sample (as was done in this investigation). It is essential to note, that the x100 oil-immersed objective we used has the correction (lowest aberrations) at the depth of $170 \mu \mathrm{m}$ as well. We believe, that the combination of two factors, namely, thorough optimisation of the inscription conditions when we position the periodic structure at the depth of the best resolution of the monitoring objective, enabled us to achieve the lowest

The minimal pulse energy value of $30 \mathrm{~nJ}$ used for the inscription can be compared with the typical pulse energy value of $600 \mathrm{~nJ}$ used earlier in $800 \mathrm{~nm}$ nanostructure fabrication [10, 11]. Such a decrease in the inscription energy is in line with the decrease of the order of the absorption process, from five-photon to two-photon $[14,17]$. It should be emphasized that the laser pulse peak power values used in our experiments $(100-300 \mathrm{~kW})$ correspond to the critical power for self-focusing in fused silica, which at $267 \mathrm{~nm}$ is estimated to be $\sim 150 \mathrm{~kW}$ (compared with $2300 \mathrm{~kW}$ at $800 \mathrm{~nm}$ [11]). The importance of the $170-\mu \mathrm{m}$-thick glass layer for the substantial decrease of the focal beam spot is undoubtedly related to nonlinear effects. 


\section{Numerical modelling}

For numerical modeling of the plasma formation during nonlinear propagation of highintensity femtosecond $267 \mathrm{~nm}$ laser pulse through fused silica media with subsequent twophoton absorption (TPA) and self-focusing, the adaptive mesh approach [20, 21] was used. This approach was already used earlier for the theoretical description of the five-photon absorption case, which takes place while the high-intensity $800 \mathrm{~nm}$ femtosecond pulse is propagating through the same medium $[10,11]$. Here we will present only some essential results of numerical calculations for UV PbP inscription, the detailed description will be published elsewhere.

First we will determine two important physical parameters for $267 \mathrm{~nm}$ case, one of them being the critical self-focusing power

$$
P_{c r}=\frac{\lambda^{2}}{2 \pi n n_{2}}
$$

where $\lambda$ is the inscription wavelength, $n$ is the linear refractive index at the inscription wavelength [22] and $n_{2}$ is the nonlinear refractive index which is for fused silica nearly constant through the high transparency region and equal to $3 \times 10^{-16} \mathrm{~cm}^{2} / \mathrm{W}$ [22]. The calculation made gives a value of about $250 \mathrm{~kW}$. This is by an order of magnitude smaller than the similar value for the $800 \mathrm{~nm}$ case $(2300 \mathrm{~kW}[11,20])$. It should also be emphasized that in our case of recording the structures with the $250 \mathrm{~nm}$ period $(\sim 125 \mathrm{~nm}$ spot diameter), it means we are reaching the intensity of $2 \times 10^{15} \mathrm{~W} / \mathrm{cm}^{2}$.

Another important parameter is the $I_{t h}$ "threshold intensity" for femtosecond inscription to begin [2]. It originates from the rate equation for plasma generation under femtosecond laser radiation:

$$
I_{t h}=\left(\frac{K \cdot \hbar \omega \cdot \rho_{B D}}{\tau_{P} \cdot \beta^{(K)}}\right)^{1 / K}
$$


the multiphoton absorption coefficient $\beta^{(K)}$ can be expressed as: $\beta^{(K)}=\hbar \omega \cdot \sigma_{K} \cdot \rho_{A T}$, with the $\rho_{A T}=2.1 \cdot 10^{22} 1 / \mathrm{cm}^{3}$ is the density of atoms, and $\sigma_{K}$ with $K=2$ for two photon excitation (Eg $=$ $7.6 \mathrm{eV}$ is assumed in fused silica) is the two-photon absorption cross-section, $\rho_{B D}$ is the plasma frequency $\left(\rho_{\mathrm{BD}} \sim 1.6 \cdot 10^{22} 1 / \mathrm{cm}^{3}\right)$. The estimation using formula (2) with $\beta_{2}=2 \cdot 10^{-11} \frac{\mathrm{cm}}{\mathrm{W}}$ gives the threshold of femtosecond inscription at $267 \mathrm{~nm}$ of about $6.2 \cdot 10^{13} \mathrm{~W} / \mathrm{cm}^{2}$. This is more than two times higher than the threshold intensity at $800 \mathrm{~nm}\left(2.5 \cdot 10^{13} \mathrm{~W} / \mathrm{cm}^{2}\right)$.

The laser peak power in our experiments with UV femtosecond laser was of the order of $P_{c r}$. Top margin of the intensity for UV femtosecond micro-fabrication may be obtained with the following expression for $I_{T P A}$ - the saturation intensity of TPA-induced ionisation:

$$
I_{T P A}=\sqrt{\frac{1}{\sigma_{2} \tau_{e}}}=\sqrt{\frac{h v \rho_{a t}}{\beta_{2} \tau_{e}}}
$$

where $\sigma_{2}$ is the absorption cross-section of two-photon transition, $\tau_{\mathrm{e}}=1.7 \times 10^{-15} \mathrm{~s}$ is the electronic collision relaxation time, $h v$ - photon energy at $267 \mathrm{~nm}, \rho_{\mathrm{at}}=2.1 \times 10^{22}$ atoms $/ \mathrm{cm}^{3}$ is the material concentration, $\beta_{2}=2 \cdot 10^{-11} \mathrm{~cm} / \mathrm{W}$ is the two-photon absorption coefficient at this wavelength $[22,23]$. The calculations give for $I_{T P A}$ a value of $7 \cdot 10^{14} \mathrm{~W} / \mathrm{cm}^{2}$ at $267 \mathrm{~nm}$ irradiation, which is by more than order of magnitude higher than the value obtained above with the formula (2).

Based on the results of the numerical simulation we found that the intensity could not reach $I_{T P A}$ $\left(7 \cdot 10^{14} \mathrm{~W} / \mathrm{cm}^{2}\right)$ regardless of the input laser pulse energy power and inscription depth.

As it was mentioned in [24], the ratio of critical power for self-focusing and the threshold intensity for inscription defines a "critical" focal spot area:

$$
\frac{P_{c r}}{I_{T H}}=S_{C R}
$$


The significance of this parameter is in the following: if the focal spot is larger than $S_{C R}$ (linear focusing), the Kerr nonlinear term will contribute to the dynamics of focusing. Estimation using formula (3) gives critical radius of the order of $360 \mathrm{~nm}$. As we mentioned above, in our experiment we expect the focal spot radius to be of about $250-300 \mathrm{~nm}$, which is close to critical radius. This means that we have to consider the contribution of nonlinear effects. Using the formula (5) from [24] we can estimate the threshold energy for femtosecond inscription to be 5nJ only (due to the Kerr nonlinearity). The 6 times difference with our experiment may be attributed to the non-Gaussian profile of the intensity distribution in our experiment due to the specific design of our objective.

Figures 8 a, b, c, d, e demonstrate the plasma density distributions (normalized to plasma breakdown density) in fused silica for the focusing depth of $200 \mu \mathrm{m}$ and five different values of laser radiation power, from $0.2 P_{\mathrm{cr}}$ to $5 P_{\mathrm{cr}}$. It is clearly seen how, with the raise of intensity, the self-focusing takes place and the "light bullet" forms. Figures 9 a, b, c demonstrate the plasma density distributions for three different focusing depths (100, 200 and $300 \mu \mathrm{m})$ and radiation power of $5 P_{\mathrm{cr}}$. It is evident that the focusing depth of $200 \mu \mathrm{m}$ is optimal, as it corresponds to the smallest beam cross-section value. Hence the results of numerical calculations are in good agreement with our experimental findings.

\section{Conclusion}

Using $30 \mathrm{~nJ}, 267 \mathrm{~nm}, 300$ fs laser pulses, tightly-focussed at a depth of $170 \mu \mathrm{m}$ below the surface of a fused silica sample, we have succeeded in recording nanostructures with periods from $1000 \mathrm{~nm}$ to $250 \mathrm{~nm}$. To the best of our knowledge, the latter period has never been achieved before from this type of inscription process. The optimisation of the fused silica layer thickness led us also to the inscription of the nanostructures with an improved quality. The performed numerical modeling confirms the obtained experimental results. 


\section{Acknowledgements}

This work was funded by EPSRC (Grant EP/D060990/1). David N. Nikogosyan wishes to thank the Leverhulme Trust for his Visiting Professorship Award (Ref. F002250G). Padraig Bolger and Anatoly V. Zayats acknowledge the support from the EC FP6 project PLASMOCOM.

\section{Reference list}

1. E. Wikszak, J. Burghoff, M. Will, S. Nolte, A. Tünnermann, T. Gabler, In: Conference on Lasers and Electro-Optics, 16-21 May 2004, San-Francisco, USA, OSA Trends in Optics and Photonics, 96, paper CThM7 (2004)

2. A. Martinez, M. Dubov, I. Khrushchev, I. Bennion, In: Conference on Lasers and ElectroOptics CLEO/IQEC 2004, 16-21 May 2004, San-Francisco, USA, OSA Trends in Optics and Photonics 96, paper CMY6 (2004)

3. A. Martinez, M. Dubov, I. Khrushchev, I. Bennion, Electron. Lett. 40, 1170 (2004)

4. A. Martinez, I.Y. Khrushchev, I. Bennion, Electron. Lett. 41, 176 (2005)

5. Y. Lai, A. Martinez, I. Khrushchev, I. Bennion, Opt. Lett. 31, 1672 (2006)

6. A. Martinez, I. Khrushchev, I. Bennion, Opt. Lett. 31, 1603 (2006)

7. G.D. Marshall, M. Ams, M.J. Withford, Opt. Lett. 31, 2690 (2006)

8. A. Martinez, M. Dubov, I. Khrushchev, I. Bennion, IEEE Phot. Technol. Lett. 18, 2266 (2006)

9. N. Jovanovic, A. Fuerbach, G.D. Marshall, M.J. Withford, S.D. Jackson, Opt. Lett. 32, 1486 (2007)

10. M. Dubov, V. Mezentsev, I. Bennion, In: Proceedings of NSTI Nanotechnology Conference Nanotech 2006, 7-11 May 2006, Boston, USA, 3, 229 (2006) 
11. V. Mezentsev, J. Petrovic, M. Dubov, I. Bennion, J. Dreher, H. Schmitz, R. Grauer, Proc. SPIE 6459, paper 64590B (2007)

12. I. Bennion, V. Mezentsev, M. Dubov, D.N. Nikogosyan, J. Petrovic, Y. Lai, G. Smith, K. Zhou, K. Sugden, In: Proceedings of OSA Topical Meeting on Bragg Gratings Photosensitivity and Poling in Glass Waveguides BGPP 2007, 2-6 Sep. 2007, Quebec, Canada, paper BWB6

13. C.W. Smelser, S.J. Mihailov, D. Grobnic, Opt. Express 13, 5377 (2005)

14. D.N. Nikogosyan, Meas. Sci. Technol. 18, R1 (2007)

15. D-.L. Wang, C-.D. Li, L. Luo, H. Yang Q-.H. Gong, Chin. Phys. Lett. 18, 65 (2001)

16. M. Dubov, V. Mezentsev, I. Bennion, D.N. Nikogosyan, Meas. Sci. Technol. 18, L15 (2007)

17. V. Kudriasov, D. Majus, V. Sirutkaitis, S.A. Slattery, D.N. Nikogosyan, Opt. Commun. 271, $408(2007)$

18. D.N. Nikogosyan, Nonlinear Optical Crystals: A Complete Survey (Springer, New York, 2005)

19. R. Wiesendanger, Scanning Probe Microscopy (Springer, New York, 1998)

20. V. Mezentsev, J. Petrovic, J. Dreher, R. Grauer, Proc. SPIE 6107, 61070R-1 (2006)

21. J.S. Petrovic, V. Mezentsev, H. Schmitz, I. Bennion, Opt. Quant. Electron. 39, 939 (2007)

22. D.N. Nikogosyan, Properties of Optical and Laser-Related Materials. A Handbook (John Wiley, Chichester, 1997)

23. A. Dragomir, J.G. McInerney, D.N. Nikogosyan, Appl. Opt. 41, 4365 (2002)

24. S. K. Turitsyn, V. K. Mezentsev, M. Dubov, A. M. Rubenchik, M. P. Fedoruk, and E.V. Podivilov, Opt. Express 1514750 (2007). 


\section{Figure captions}

Figure 1. (a) Experimental set-up for third-harmonic generation; (b) PbP inscription set-up.

Figure 2. DIC microphotograph of the 1000-nm-period nanostructure, fabricated on the surface of a fused silica sample. The energy of the inscribing pulses was $38 \mathrm{~nJ}$. The size of the bar corresponds to $10 \mu \mathrm{m}$.

Figure 3. (a) Topography of the same 1000-nm-period nanostructure as in Fig. 2, investigated by AFM; (b) Cross-section of the image, presented in Fig. 3 a, along the inscription direction. (c) Cross-section of the image, presented in Fig. 3 a, perpendicular to the inscription direction.

Figure 4. (a) DIC microphotographs of structures with 1000, 500 and $400 \mathrm{~nm}$ periods inscribed at the optimal focusing depth of $170 \mu \mathrm{m}$ with sample translation speeds of $1.0,0.5$ and $0.4 \mathrm{~mm} / \mathrm{s}$, respectively. The energy of the inscribing pulses was $30 \mathrm{~nJ}$. The size of the bar corresponds to 10 $\mu \mathrm{m}$. (b) DIC microphotographs of similar structures inscribed earlier at a focus depth of $300 \mu \mathrm{m}$ [16]. The energy of the inscribing pulses was $82 \mathrm{~nJ}$. The size of the bar corresponds to $10 \mu \mathrm{m}$. Figure 5. DIC microphotographs of structures with 300 and $250 \mathrm{~nm}$ periods inscribed at the optimal focus depth of $170 \mu \mathrm{m}$ with sample translation speeds of 0.3 and $0.25 \mathrm{~mm} / \mathrm{s}$, 
respectively. The energy of the inscribing pulses was $30 \mathrm{~nJ}$. The size of the bar corresponds to 10 $\mu \mathrm{m}$.

Figure 6. DIC microphotographs of structures with 1000 and $500 \mathrm{~nm}$ periods inscribed at a focus depth of $330 \mu \mathrm{m}$ with sample translation speeds of 1.0 and $0.5 \mathrm{~mm} / \mathrm{s}$, respectively. The energy of the inscribing pulses was $90 \mathrm{~nJ}$. The size of the bar corresponds to $10 \mu \mathrm{m}$.

Figure 7. DIC microphotographs of structures with 400 and $300 \mathrm{~nm}$ periods inscribed at the optimal focus depth of $170 \mu \mathrm{m}$ with sample translation speeds of 0.4 and $0.3 \mathrm{~mm} / \mathrm{s}$, respectively. The energy of the inscribing pulses was $90 \mathrm{~nJ}$. The size of the bar corresponds to $10 \mu \mathrm{m}$.

Figure 8. Plasma density distribution (normalized to plasma breakdown density) in a fused silica for the focusing depth of $200 \mu \mathrm{m}$ and different laser radiation power values: (a) $0.2 P_{\mathrm{cr}}$; (b) 0.5 $P_{\mathrm{cr}}$; (c) $1.0 P_{\mathrm{cr}}$; (d) $2.0 P_{\mathrm{cr}}$; (e) $5 P_{\mathrm{cr}}$. The divisions on vertical and horizontal axes are in mm, they represent the propagation direction and the perpendicular one, respectively. The colour scale corresponds to plasma density distribution normalized to plasma breakdown density.

Figure 9. Plasma density distribution (normalized to plasma breakdown density) in a fused silica for laser radiation power of $5 P_{\mathrm{cr}}$ and different focusing depths: (a) $100 \mu \mathrm{m}$; (b) $200 \mu \mathrm{m}$; (c) 300 $\mu \mathrm{m}$. The divisions on vertical and horizontal axes are in $\mathrm{mm}$, they represent the propagation direction and the perpendicular one, respectively. The colour scale corresponds to plasma density distribution normalized to plasma breakdown density. 


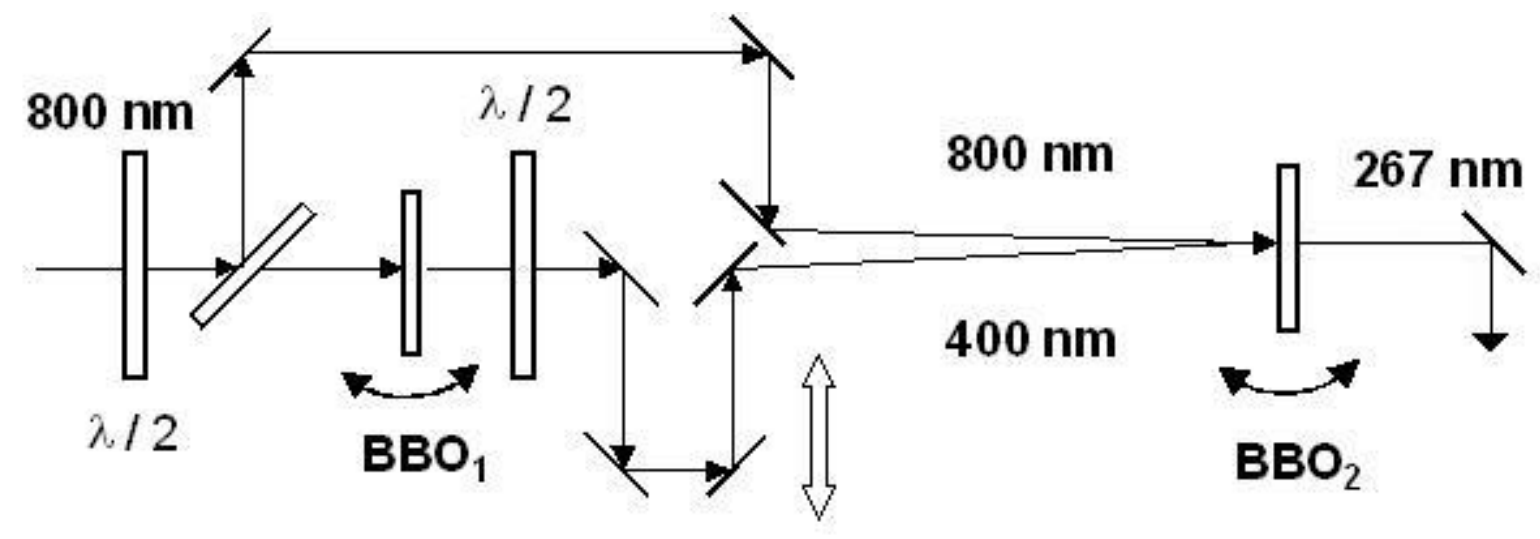

a) 
Figure 1 a

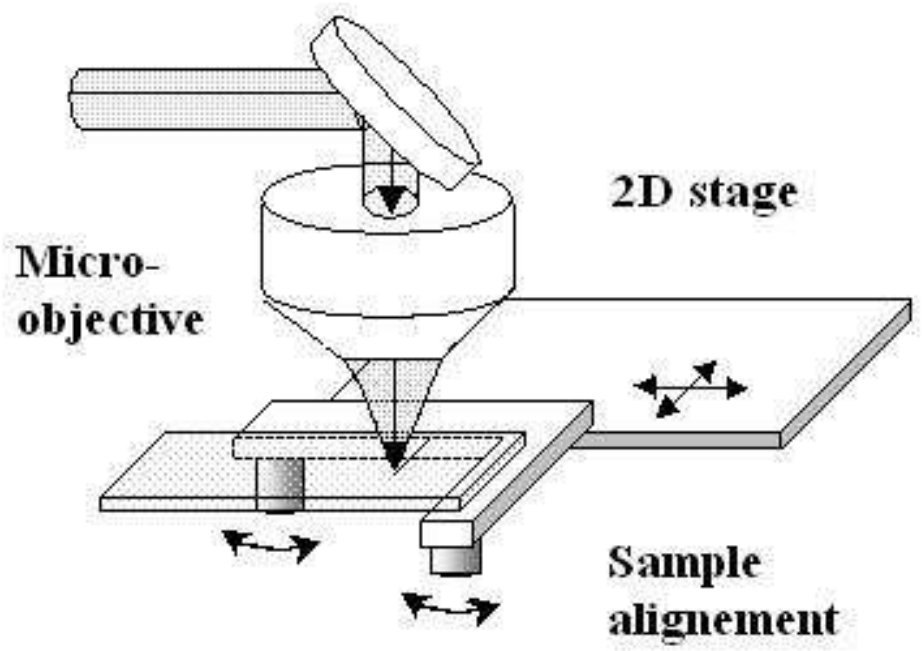

b) 
Figure 1 b

\section{$1.0 \mathrm{~mm} / \mathrm{s}$}

\section{$10 \mu \mathrm{m}$}


Figure 2

\section{$1.0 \mathrm{~mm} / \mathrm{s}$}

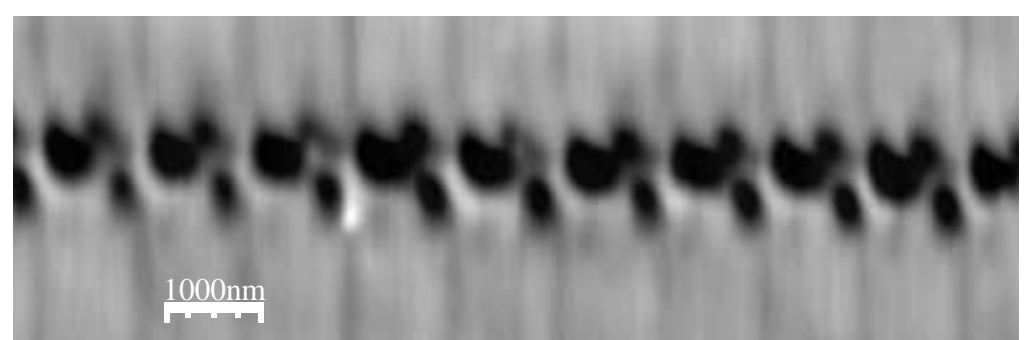

a) 
Figure 3a

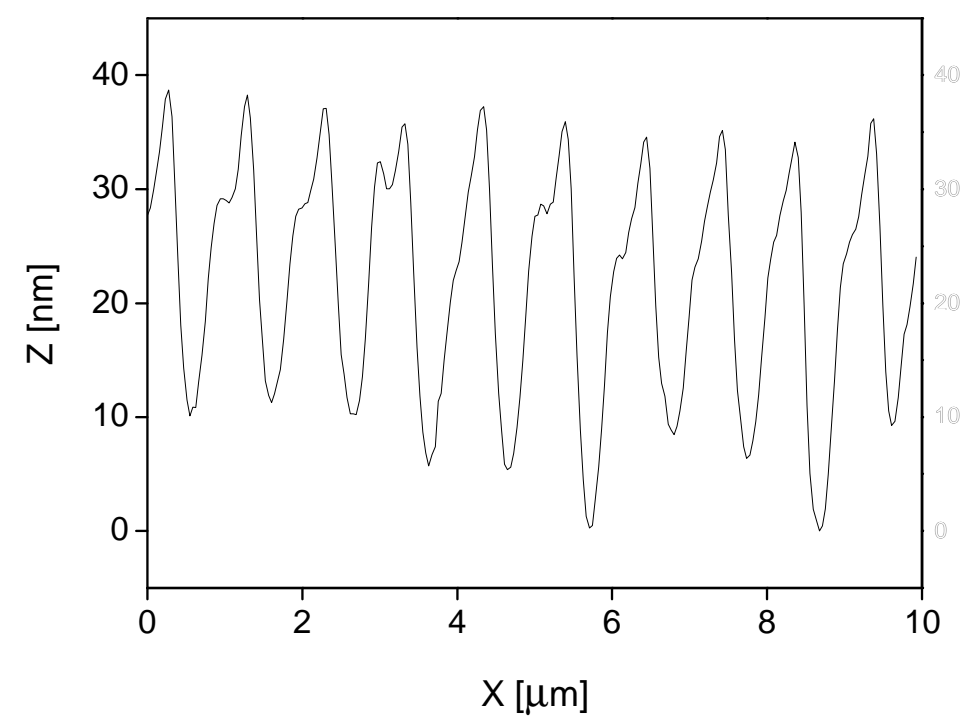

b)

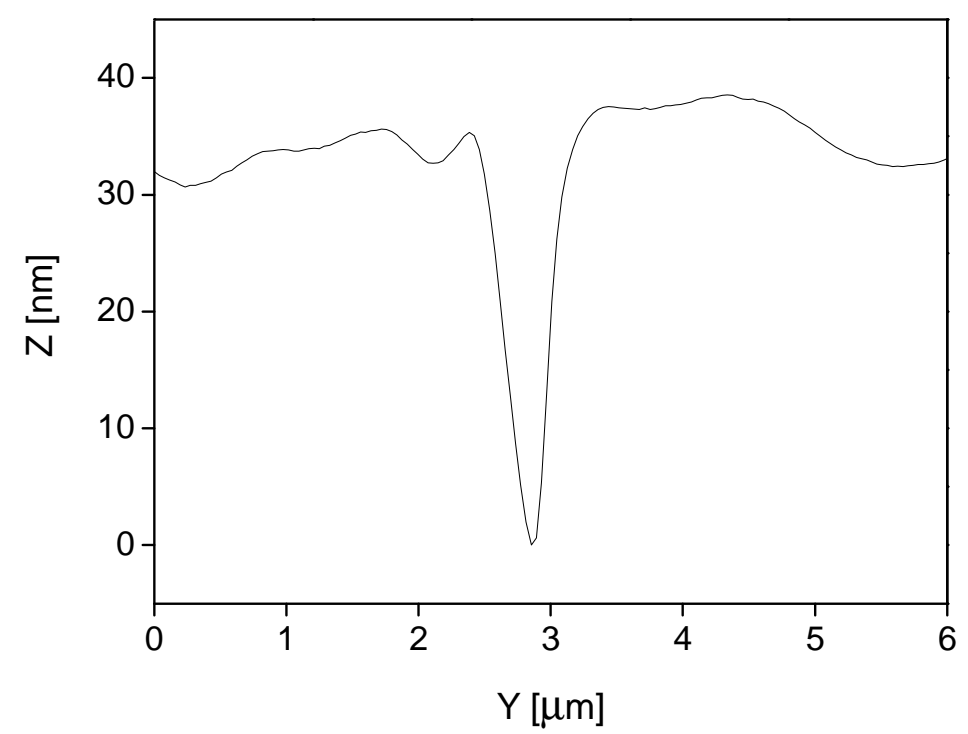


c)

Figure 3 b, c

\section{$1.0 \mathrm{~mm} / \mathrm{s}$}

\section{$0.5 \mathrm{~mm} / \mathrm{s}$}

$0.4 \mathrm{~mm} / \mathrm{s}$

\section{$10 \mu \mathrm{m}$}

a) 
Figure 4 a

\section{$1.0 \mathrm{~mm} / \mathrm{s}$}

$0.5 \mathrm{~mm} / \mathrm{s}$

$0.4 \mathrm{~mm} / \mathrm{s}$

$10 \mu \mathrm{m}$

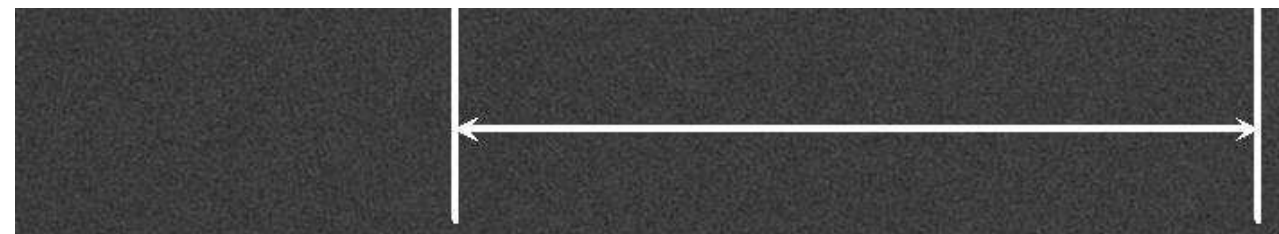


b)

Figure 4 b

\section{$0.3 \mathrm{~mm} / \mathrm{s}$}

$0.25 \mathrm{~mm} / \mathrm{s}$

\section{$10 \mu \mathrm{m}$}


Figure 5

\section{$1.0 \mathrm{~mm} / \mathrm{s}$}

$0.5 \mathrm{~mm} / \mathrm{s}$

\section{$10 \mu \mathrm{m}$}


Figure 6

\section{$0.4 \mathrm{~mm} / \mathrm{s}$}

\section{$0.3 \mathrm{~mm} / \mathrm{s}$}

$10 \mu \mathrm{m}$

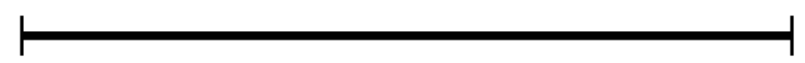


Figure 7

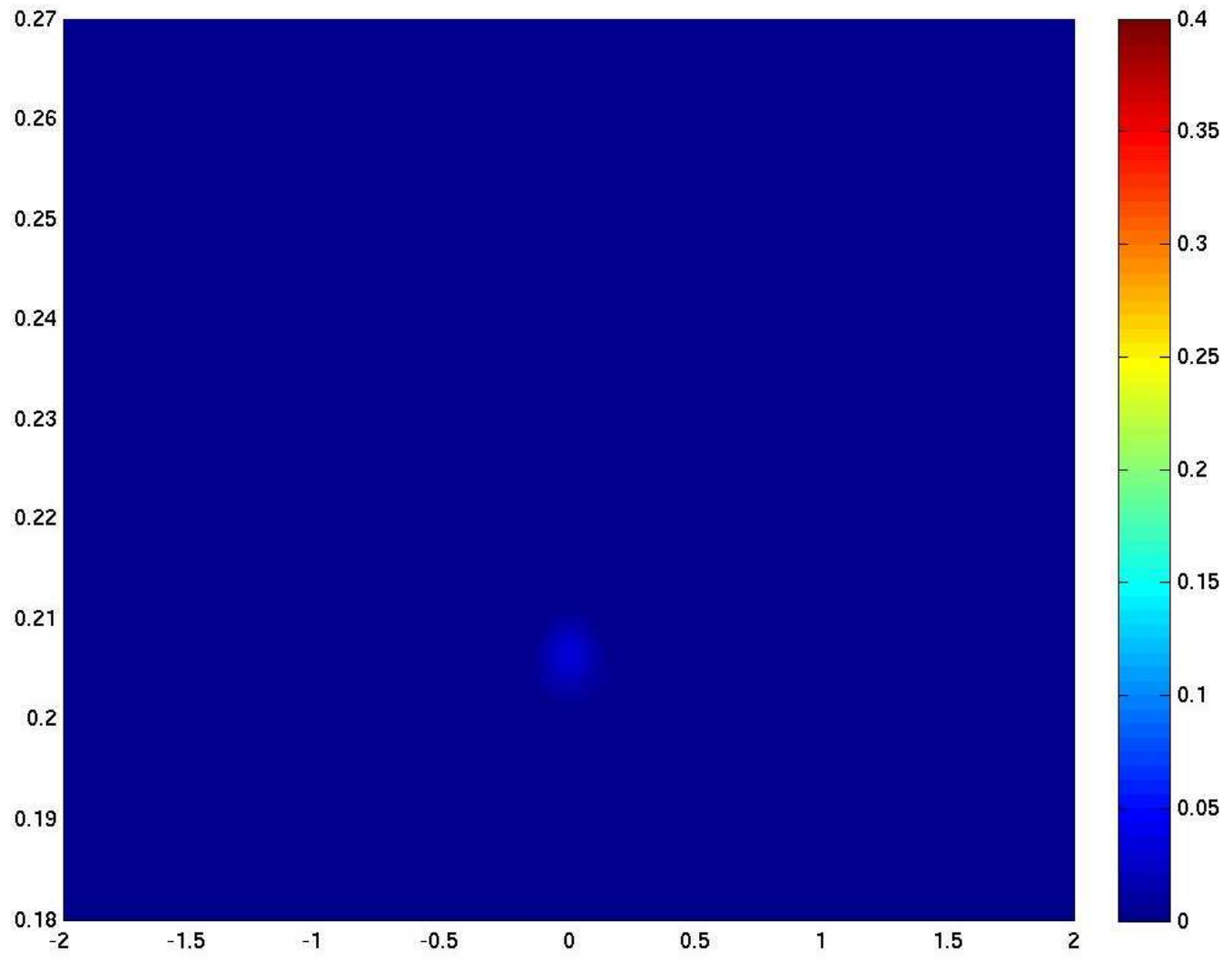

Figure 8a 


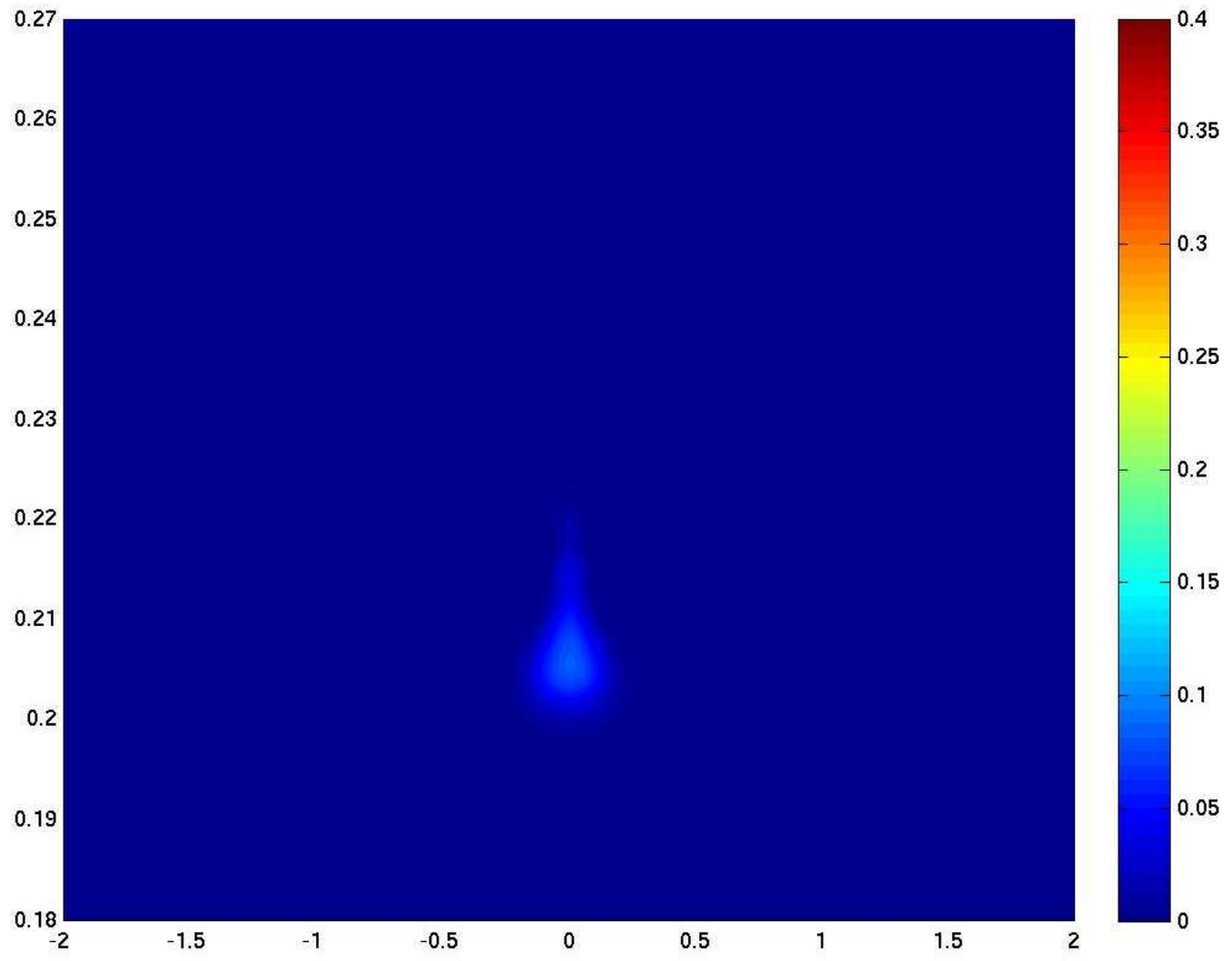

Figure $8 b$ 


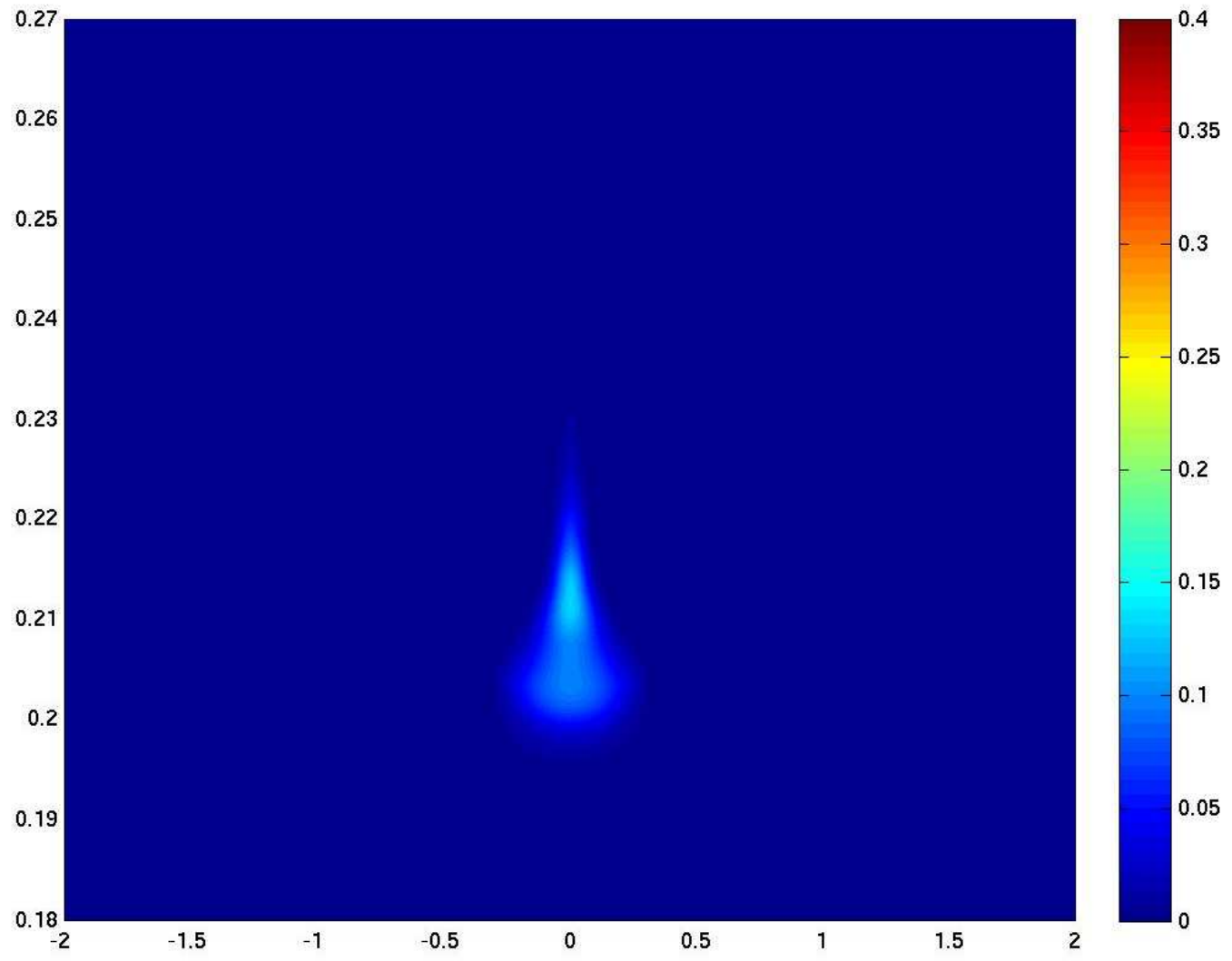

Figure 8c 


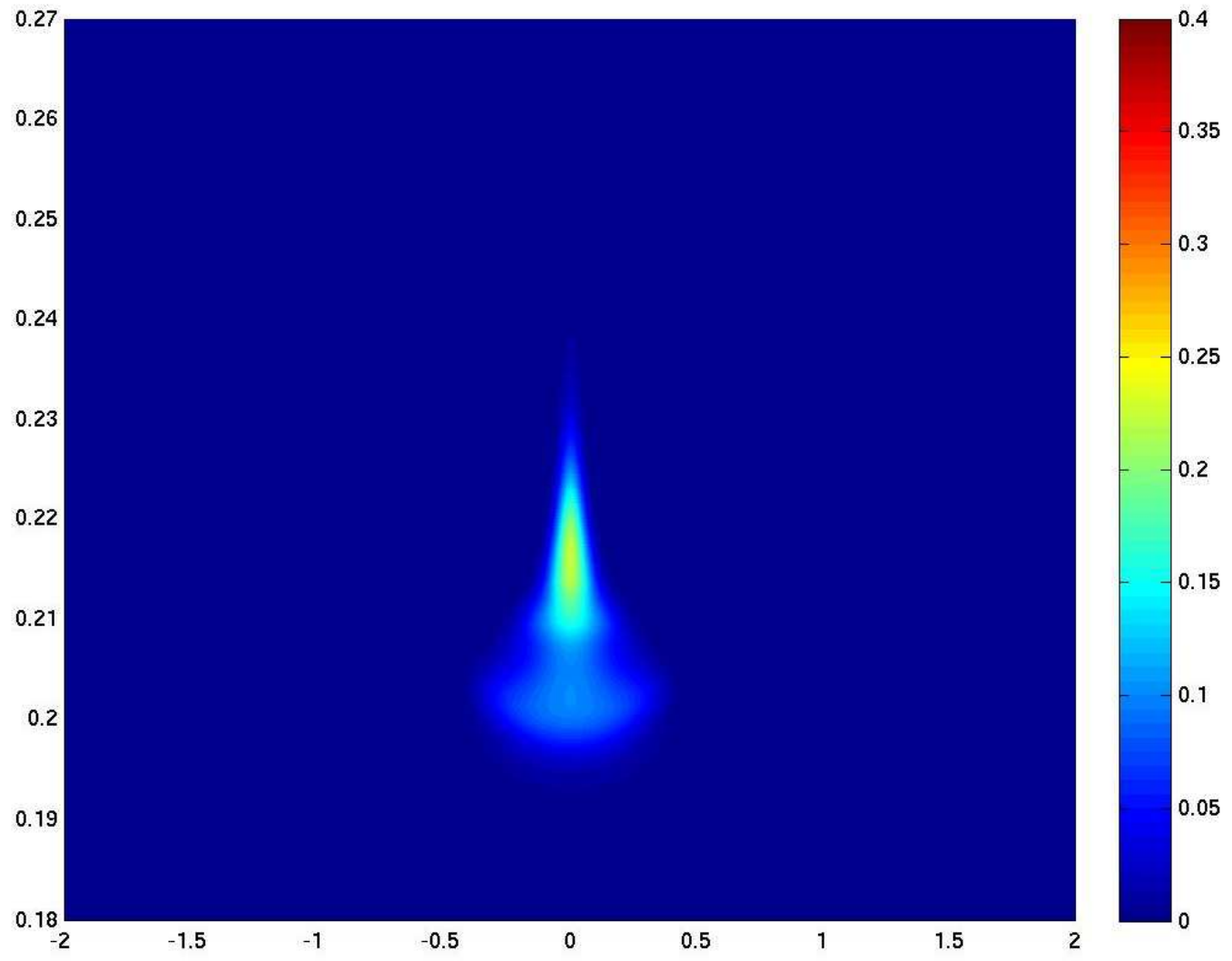

Figure 8d 


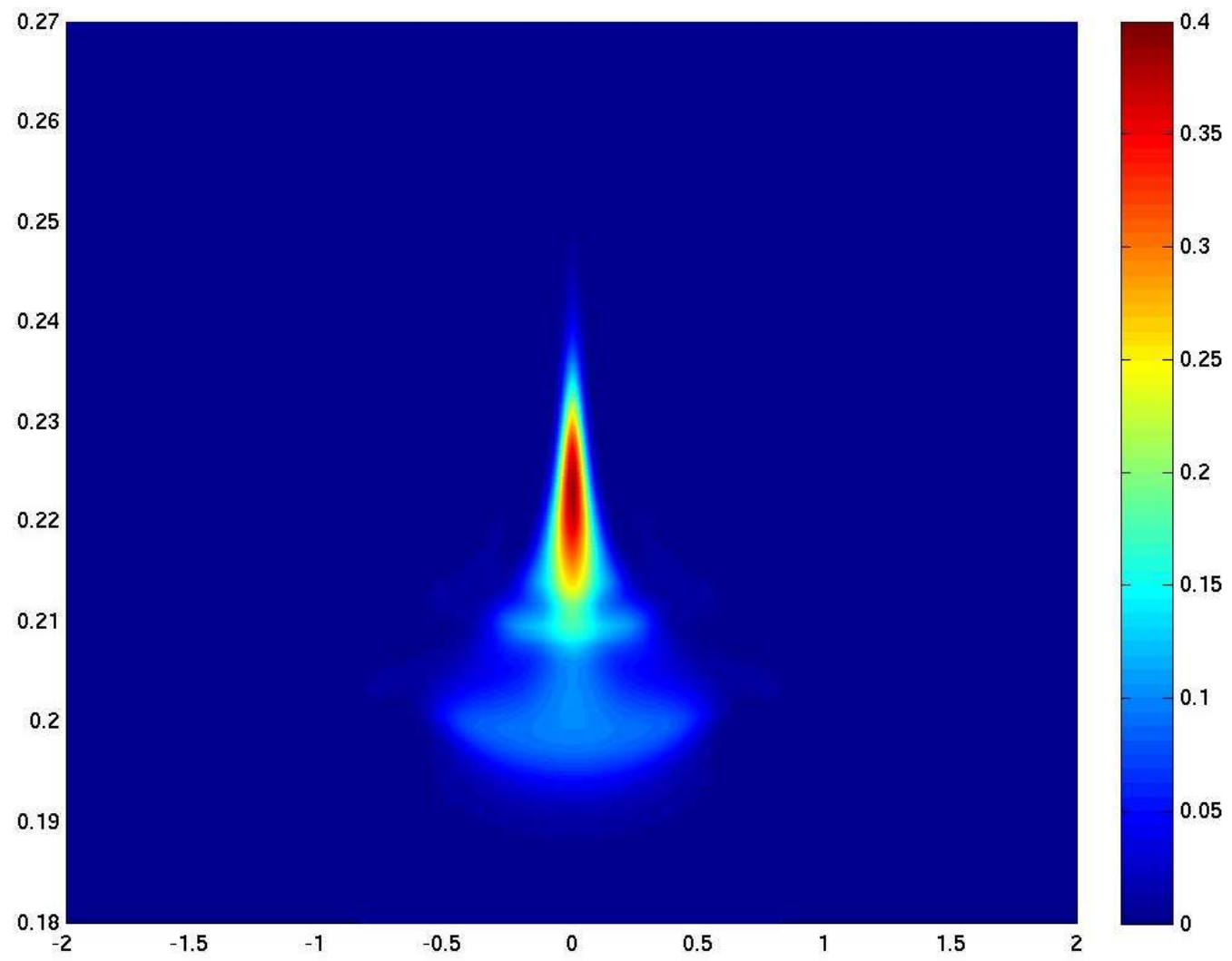

Figure 8e 


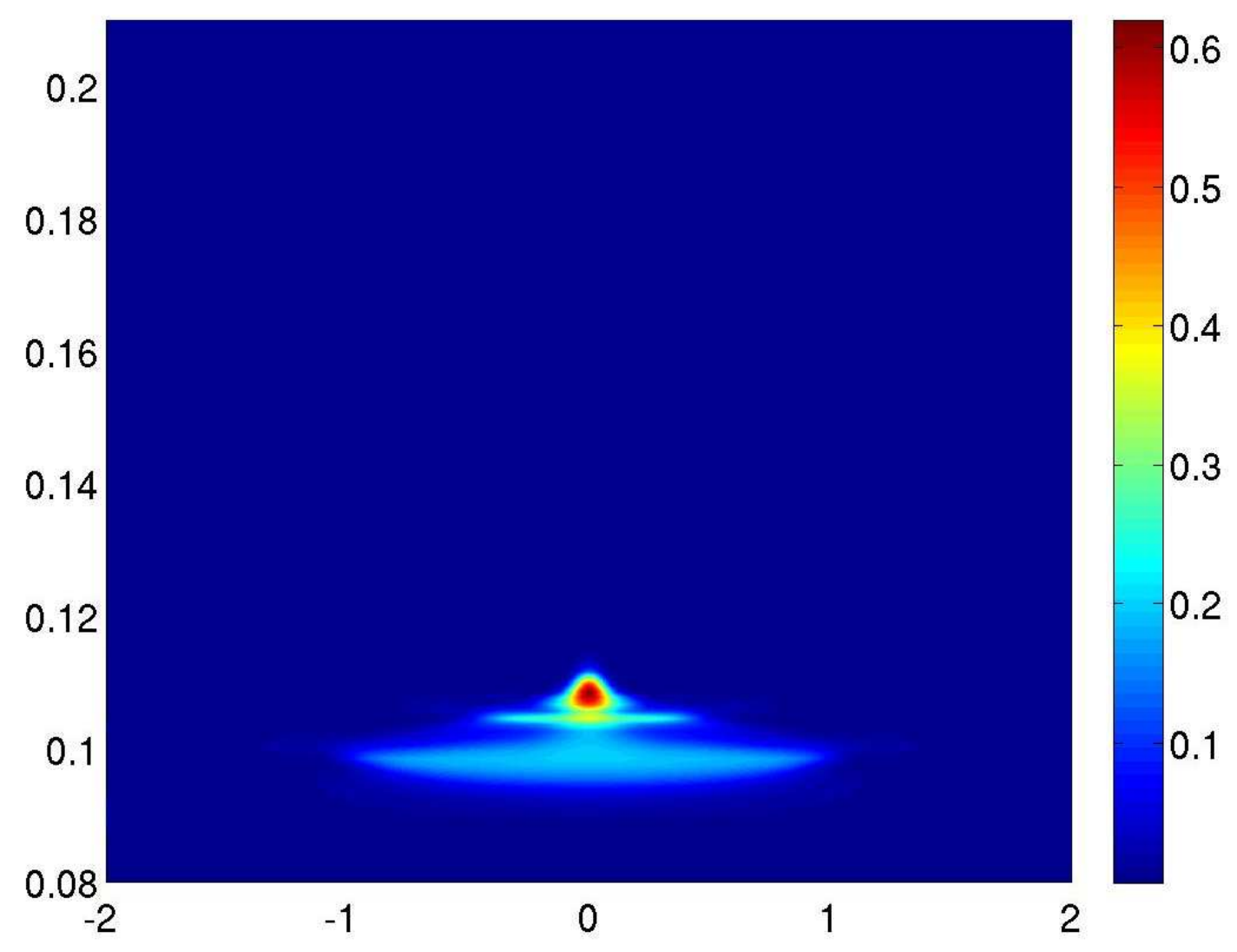

Figure 9a 


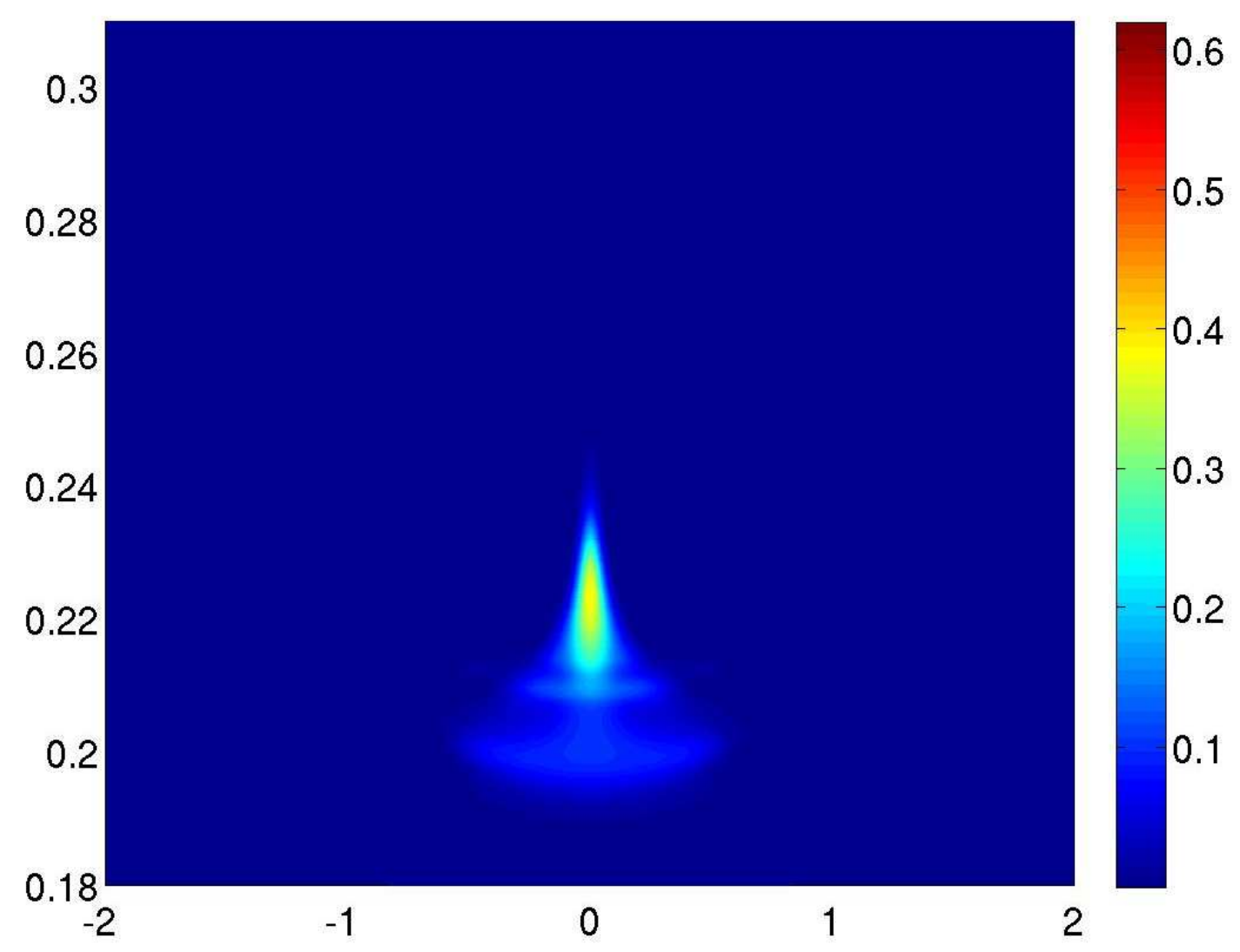

Figure 9b 


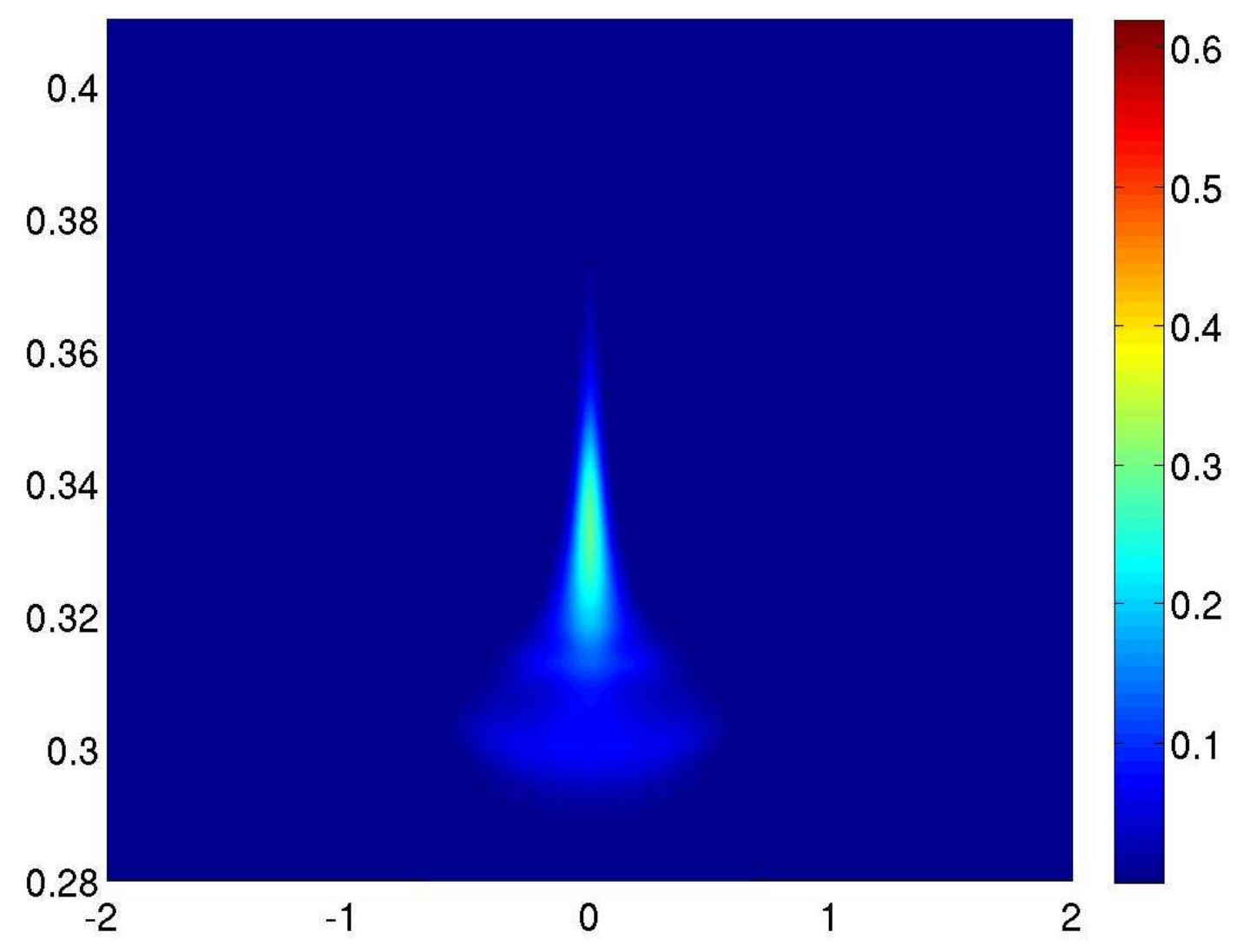

Figure 9c 